\title{
Parent Artery Occlusion in Large, Giant, or Fusiform Aneurysms of the Carotid Siphon: Clinical and Imaging Results
}

\author{
M.-A. Labeyrie, S. Lenck, D. Bresson, J.-P. Desilles, A. Bisdorff, J.-P. Saint-Maurice, and E. Houdart
}

\begin{abstract}
BACKGROUND AND PURPOSE: Parent artery occlusion has long been considered the reference treatment for large/giant or fusiform aneurysms of the carotid siphon. However, meager recent data exist on this technique, which tends to be replaced by stent-assisted reconstructive techniques. In our department since 2004, we have assessed the safety, efficacy, and complication risk factors of parent artery occlusion by using coils for trapping these aneurysms.
\end{abstract}

MATERIALS AND METHODS: We determined retrospectively the complication rate, factors associated with the occurrence of an ischemic event, changes in symptoms of mass effect, evolution of the aneurysmal size, and the growth of an additional aneurysm after treatment.

RESULTS: Fifty-six consecutive patients were included, with a median age of 54 years (range, 25-85 years; 92\% women), 48\% with giant aneurysms and $75 \%$ with infraclinoid aneurysms. There was a permanent morbidity rate of $5 \%$ exclusively due to ischemia, a zero mortality rate, an aneurysmal retraction rate of $91 \%$, and an improvement rate for pain of $98 \%$ and for cranial nerve palsy of $72 \%$, with a median follow-up of $>3$ years. Para-/supraclinoid topography of the aneurysm $(P=.043)$ and the presence of cardiovascular risk factors $(P=.024)$ were associated with an excessive risk of an ischemic event, whereas the presence of a mural thrombus had a protective role $(P=.033)$.

CONCLUSIONS: In this study, parent artery occlusion by using coils to treat large/giant or fusiform aneurysms of the carotid siphon was safe and effective, especially for giant infraclinoid aneurysms. According to recent meta-analyses, these results suggest that the validation of stent-assisted reconstructive treatments for these aneurysms requires controlled studies with parent occlusion artery.

ABBREVIATIONS: BOT = balloon occlusion test; $\mathrm{IE}=$ ischemic event; $\mathrm{PAO}=$ parent artery occlusion; $\mathrm{Q}$-Q3 = interquartile range

$\mathbf{T}$ here is a high risk of severe bleeding, debilitating pain, or progressive cranial nerve palsy with symptomatic, intradural, or some extradural, large, giant, or fusiform aneurysms of the carotid siphon. ${ }^{1-3}$ The selective endovascular coiling of this type of aneurysm is not usually adopted due to the low rate of recovery and technical difficulties when the neck of the aneurysm is large. ${ }^{4-6}$ The surgical treatment (selective or deconstructive by trapping) is technically challenging and is rarely performed, in our experience, for large supracavernous aneurysms with a well-tolerated occlusion test, and it is usually not performed for large intracavernous aneurysms because of the risk of cranial nerve injury. Proximal occlusion without trapping (surgical or endovascular) has a lower rate of aneurysmal retraction and should not be performed for carotid aneurysms.

Received March 24, 2014; accepted after revision June 23.

From the Departments of Interventional Neuroradiology (M.-A.L., S.L., J.-P.D., A.B., J.-P.S.-M., E.H.) and Neurosurgery (D.B.), Université Paris Diderot, Pôle Neurosensoriel, Lariboisière Hospital, Paris, France.

Please address correspondence to Marc-Antoine Labeyrie, MD, Service de Neuroradiologie, Hôpital Lariboisière, 2 Rue Ambroise Paré, 75010, Paris, France;

e-mail: marc-antoine.labeyrie@lrb.aphp.fr

http://dx.doi.org/10.3174/ajnr.A4064
Endovascular parent artery occlusion (PAO) with trapping of the aneurysm has long been considered the reference treatment for large, giant, or fusiform aneurysms of the carotid siphon. However, data from the literature assessing this technique focus on small or old series using mainly detachable balloons, which are no longer available in most departments of neuroradiology. ${ }^{7-11}$ Besides, developing new reconstructive stent-assisted embolization techniques, including flow diversion, offer a therapeutic possibility for this type of aneurysm that could be preferred to deconstructive techniques. ${ }^{12-14}$ Assessing carotid occlusion for treating this type of aneurysm remains relevant today.

The main objective of this study was to assess the safety and efficacy of PAO by using coils to treat large, giant, or fusiform aneurysms of the carotid siphon. The secondary objective was to determine the factors associated with the occurrence of an ischemic event (IE) in the outcomes.

\section{MATERIALS AND METHODS Patients}

From a prospective registry, we retrospectively included consecutive patients treated in our center between January 2004 and 
December 2013 by PAO for a saccular aneurysm with a diameter of $\geq 15 \mathrm{~mm}$ or a fusiform aneurysm (regardless of its diameter) on the carotid siphon. Indications for PAO for these kinds of aneurysms were limited, in our center, to intradural aneurysms and/or symptomatic aneurysms and/or aneurysms with sphenoid sinus erosion. Proximal carotid occlusions without trapping were excluded from the analysis. For each patient, we collected the following data: 1) age and sex, 2) the presence of at least 1 cardiovascular risk factor, 3) the administration of antiplatelet therapy during the procedure, and 4) the symptoms at presentation (asymptomatic, pain [including headache, hemifacial pain, or retro-orbital pain], cranial nerve palsy, subarachnoid hemorrhage, or cavernous carotid fistula). Cerebral imaging before the procedure was reviewed to determine the characteristics of the aneurysm: 1) its topography (infraclinoid versus para-/supraclinoid), 2) its morphology (giant saccular aneurysms [ $\geq 25 \mathrm{~mm}$ ], large saccular aneurysms [ $\geq 15 \mathrm{~mm}$ and $<25 \mathrm{~mm}$ ], and fusiform aneurysms), 3) the presence of a mural thrombus, 4) the presence of additional aneurysms, and 5) a delay of $\geq 2$ seconds on the venous filling during the balloon occlusion test (BOT).

\section{Parent Artery Occlusion}

PAO was performed in a local standardized way after a $70-\mathrm{IU} / \mathrm{kg}$ bolus of heparin. No systematic heparin was maintained afterwards. No systematic antiplatelet therapy was introduced before, during, or after the procedure. Dual antiplatelet therapy (aspirin, $75 \mathrm{mg} /$ day, and clopidogrel, $75 \mathrm{mg} /$ day) was introduced 7 days before the treatment when a reconstructive treatment was initially planned. In these cases, an antiplatelet assay was systematically performed, and the antiplatelet therapy was adjusted when necessary.

A preliminary BOT was performed by using an inflated nondetachable balloon (Magic B1; Balt, Montmorency, France; or HyperForm, 4-15 mm; Covidien, Irvine, California) in the intrapetrous segment of the internal carotid artery. The occlusion was considered well-tolerated if the relative delay of venous filling was $\leq 1$ second (Fig $1 B,-C$ ) or if it was $\leq 4$ seconds with a perfect clinical tolerance for 20 minutes, even after a hypotension test with intravenous injection of a 1- to $2-\mathrm{mg}$ bolus of nicardipine to decrease the mean arterial pressure $>20 \mathrm{~mm} \mathrm{Hg}$ compared with baseline. As in other series, a delay of $>4$ seconds was considered a contraindication to permanent occlusion of the internal carotid artery. ${ }^{15}$ The definitive occlusion of the artery was performed by trapping the aneurysm by using detachable platinum coils (Fig $1 A$ ). The objective was to completely stop the blood flow at the neck of the aneurysm. Ideally, an upstream and downstream occlusion of the aneurysm with loose packing in the aneurysm was performed. When the upstream segment was too short to set up coils, an occlusion of the aneurysm and of the parent artery was performed in opposition and downstream. The occlusion was considered satisfying if there was absolutely no progression of the contrast agent downstream of the occlusion. Occlusion was performed with soft, detachable platinum 0.014-inch-diameter coils and 30- to 50-cm length (Soft Detach-18S Embolization Coil system; Cook Medical, Bjaeverskov, Denmark). Relying on our experience, we noticed that at least 3 meters of coils were needed to occlude a carotid artery. Conventional intracranial detachable
3D-shaped 0.010-inch-diameter platinum coils were used as a first coil to occlude short downstream segments. All the procedures, including BOT and definitive occlusion, were performed as first-line with the patient under local anesthesia. They were performed with the patient under general anesthesia in noncompliant patients or when a reconstructive treatment was initially planned. After permanent occlusion, the patients were kept in the intensive care unit for at least 48 hours for monitoring, according to previously described protocol in the literature. ${ }^{15}$

Before the procedure, informed written consent was obtained from all the patients. This study was approved by the ethics committee "Ile-de-France 4."

\section{Clinical and Imaging Follow-Up}

Clinical and imaging data were retrospectively analyzed from the medical file and prospectively analyzed with a systematic phone call to patients by an interventional neuroradiologist independent of the procedures (M.-A.L.). We distinguished early ( $\leq 30$ days) or late ( $>30$ days until the last follow-up) follow-up. Patients for whom no clinical or imaging data were available for $>30$ days after the procedure were considered lost to follow-up and were excluded from the analysis of the late follow-up period.

We determined the occurrence of the following variables: 1) a hemorrhagic stroke, 2) a symptomatic cerebral or retinal IE (with resolved or persistent symptoms at discharge), 3) death regardless of its cause, 4) changes in the cranial nerve palsy intensity felt according to a qualitative scale (resolved/improved, unchanged, worsened/appearing), 5) changes in the treated aneurysm on CT or MR imaging (retracted, unchanged, or grown) (retraction of the sac was arbitrarily defined as a decrease of $>10 \%$ of the diameter of the aneurysm), and 6) the growth of an additional aneurysm (de novo or pre-existing aneurysm). Given the good correlation between BOT timing and quantitative cerebral perfusion, ${ }^{16,17}$ IEs occurring in patients with a phlebogram delay of $<2$ seconds during the occlusion test were considered embolic. We also determined the modified Rankin Scale score at the last follow-up for all included patients, whatever the follow-up time.

\section{Statistical Analysis}

Statistical analysis was performed by using SPSS, Version 15.0, software (IBM, Armonk, New York). Continuous variables were described as medians and interquartile ranges (Q1-Q3), and were compared with a Mann-Whitney-Wilcoxon test. Variables by category were compared by using a $\chi^{2}$ or Fisher exact test.

The factors associated with the occurrence of cerebral/retinal IEs after treatment were analyzed with a univariate analysis and then with an exploratory binary logistic regression (for variables with $P<.1$ in univariate analysis).

\section{RESULTS}

\section{Population}

Fifty-six consecutive patients treated by PAO for large, giant, or fusiform aneurysms of the carotid siphon were included in the study. Clinical and imaging data before the procedure were available for every patient. The patients had all been treated by using coils according to the protocol and indications previously defined. All patients had a well-tolerated BOT with a relative delay of 

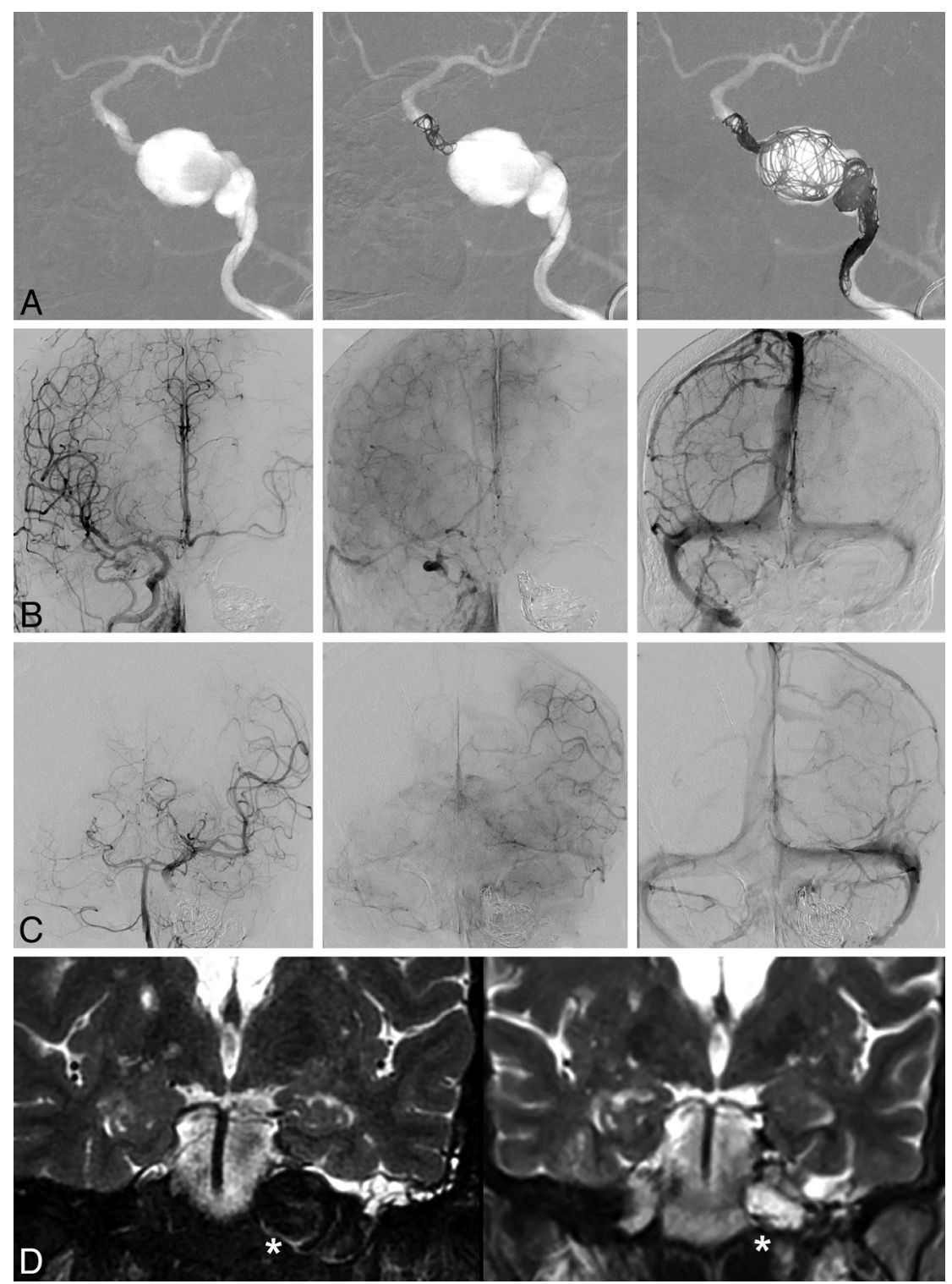

FIG 1. Parent artery occlusion in a 51-year-old woman with a left giant intrapetrous carotid aneurysm revealed by a hearing loss. Front projection of angiography before and during the occlusion $(A)$, final angiography through the right carotid artery $(B)$, final angiography through the left vertebra $(C)$, and T2 coronal MR imaging before and 2 years after treatment $(D)$. $A$, Coils were set upstream and downstream of the aneurysm, thus trapping the aneurysm and stopping the flow at its neck, and were set loosely in the aneurysm itself to maintain the thrombus. The final control $(B)$ shows that the left middle cerebral artery is partially supplied by the right carotid artery via the anterior communicating artery, but is well supplied by the vertebrobasilar axis $(C)$ via the left posterior communicating artery, with a venous return almost synchronous between the left external temporal veins and cerebellar veins. D, MR imaging shows a significant retraction of the aneurysm (white asterisk) after occlusion.

venous filling of $<4$ seconds. One patient who failed a first BOT underwent bypass surgery and had a PAO after a second BOT. A trapping with complete occlusion of the aneurysm was obtained in all patients. The patients' initial characteristics are presented in Table 1. Early clinical follow-up ( $\leq 30$ days) was available for every patient. Thirteen patients $(23 \%)$ were lost to follow-up after being discharged. Most of them (12/13) were sent and followed by hospital centers from abroad. There was no significant difference between the patients lost to follow-up and the remainder of the study population regarding the variables before treatment and the rate of early cerebral/retinal IEs in early follow-up after treatment (data not shown). For the remainder of the population, the median clinical and radiologic follow-up (Q1-Q3) was, respectively, 45 months (range, 25-90 months) and 27 months (range, 13-55 months). Among these patients, all had a clinical and MR imaging follow-up of $\geq 3$ months.

\section{Follow-Up after Carotid Occlusion}

At the last follow-up, the increase of the mRS score after the procedure was $<2$ (excellent outcome) in 53/56 (95\%) patients, equal to 2 in $2 / 56$ (3.5\%) patients (moderate aphasia and facial palsy, moderate limb paresis), and equal to 3 in 1/56 (1.5\%) patients (severe dominant arm paresis). In patients with infraclinoid aneurysms, it was $<2$ in $41 / 42$ (97.5\%) patients and equal to 2 in $1 / 42(2.5 \%)$ patients. Permanent morbidity was due exclusively to postprocedure early IEs. No intracranial hemorrhagic event or death was observed throughout the follow-up period. The outcome and the complications after treatment are presented in Table 2.

\section{Occurrence of Cerebral/Retinal IEs}

During the early follow-up, an IE was reported in $15 / 56(27 \%)$ patients and was completely resolved without sequelae at discharge in 10/15 (67\%). A good Willisian substitute (suggested by a BOT timing of $<2$ seconds) was observed in 12/15 $(80 \%)$ patients with an early IE. During the late follow-up, an IE was reported in $4 / 43(9 \%)$ patients, with a time lapse between 3 months and 2.5 years after the treatment. For all patients with a late IE, the symptoms were completely resolved at discharge. They occurred in the context of low blood pressure in 3/4 and after interruption of aspirin in 1 patient who had already had an IE during the early period. All patients with IEs were managed in a stroke unit. All had antiplatelets or heparin for the early period. Among them, 2/15 also received an intravenous bolus of abciximab (ReoPro, $10 \mathrm{mg}$ ) in the very acute phase. Patients with recurrent IEs had long-term antithrombotic therapy. The univariate analysis of the factors associated with the occurrence of an IE after treatment (whatever the delay) is presented in Table 3. The exploratory multivariate analysis (entry for $P<.1$ ) found that the existence of at least 1 cardiovascular risk factor (OR $=7$; CI 95\%, 1.3-37; $P=.024)$ and the para-/supraclinoid topography of the treated aneurysms (OR $=6$; CI 95\%, $1.1-40 ; P=.043$ ) were significantly associated with the occurrence of an IE after treatment. The presence of a mural thrombus 
Table 1: Baseline characteristics of the 56 patients of the study

\begin{tabular}{lc}
\hline \multicolumn{1}{c}{ Characteristics } & Value \\
\hline Age (yr) (median) (Q1-Q3) & $54(46-66)$ \\
Female (No.) (\%) & $51(92)$ \\
Vascular risk factor (No.) (\%) & $31(55)$ \\
Antiaggregation platelet therapy (No.) (\%) & $15(27)$ \\
Asymptomatic (No.) (\%) & $4(7)$ \\
Pain (No.) (\%) & $43(77)$ \\
Cranial nerve palsy (No.) (\%) & $40(72)$ \\
SAH or CCF (No.) (\%) & $4(7)$ \\
Giant saccular aneurysms ( $\geq 25 \mathrm{~mm})(\mathrm{No}).(\%)$ & $27(48)$ \\
Large saccular aneurysms ( $\geq 15 \mathrm{~mm}, \quad<25)($ No.) (\%) & $24(43)$ \\
Fusiform aneurysms (No.) (\%) & $5(9)$ \\
Infraclinoid aneurysms (No.) (\%) & $42(75)$ \\
Mural thrombus (No.) (\%) & $15(27)$ \\
Multiple aneurysms (No.) (\%) & $11(20)$ \\
Timing of BOT $\geq 2$ sec (No.) (\%) & $7(13)$ \\
General anesthesia (No.) (\%) & $16(28)$ \\
\hline
\end{tabular}

Note:-CCF indicates carotid cavernous fistula.

Table 2: Outcome and complications in 56 patients after carotid occlusion with coils for large, giant, or fusiform aneurysms of the carotid siphon

\begin{tabular}{|c|c|c|}
\hline Characteristics & $\begin{array}{c}\text { Early FU } \\
\text { ( } \leq 30 \text { days) } \\
(n=56)\end{array}$ & $\begin{array}{l}\text { Late FU } \\
\text { (>30 days) } \\
(n=43)\end{array}$ \\
\hline Death (No.) (\%) & 0 & 0 \\
\hline $\begin{array}{l}\text { Intracranial hemorrhage (No.) (\%) } \\
\text { Ischemic event }\end{array}$ & 0 & 0 \\
\hline Resolved symptoms (No.) (\%) & $10(18)$ & $4(9)$ \\
\hline Minor persistent symptoms ${ }^{a}$ (No.) (\%) & $2(3.5)$ & 0 \\
\hline Major persistent symptoms ${ }^{b}$ (No.) (\%) & $3(5.5)$ & 0 \\
\hline \multicolumn{3}{|l|}{ Pain } \\
\hline Resolved or improved (No.) (\%) & NA & $42(98)$ \\
\hline Unchanged (No.) (\%) & NA & 0 \\
\hline Worsened (No.) (\%) & NA & $1(2)$ \\
\hline \multicolumn{3}{|l|}{ Cranial nerve palsy } \\
\hline Resolved or improved (No.) (\%) & NA & $23^{c}(72)$ \\
\hline Unchanged (No.) (\%) & NA & $9^{c}(28)$ \\
\hline Worsened (No.) (\%) & NA & 0 \\
\hline \multicolumn{3}{|l|}{ Embolized aneurysm } \\
\hline Retracted (No.) (\%) & NA & $39(91)$ \\
\hline Unchanged (No.) (\%) & NA & $4(9)$ \\
\hline Grown (No.) (\%) & NA & 0 \\
\hline $\begin{array}{c}\text { De novo/additional growing } \\
\text { aneurysm (No.) (\%) }\end{array}$ & NA & $1(2)$ \\
\hline
\end{tabular}

Note:-NA indicates not assessed.

${ }^{\text {a }}$ Modified Rankin Scale score $\leq 1$.

${ }^{\mathrm{b}}$ Modified Rankin Scale score $>1$.

${ }^{c} n=32$.

decreased this occurrence significantly ( $O R=0.1$; CI 95\%, 0.01$0.8 ; P=.033)$. The other variables were not significantly associated with the occurrence of an IE: age, fusiform nature of the aneurysm, aneurysm size of $\geq 25 \mathrm{~mm}$, and the administration of antiplatelet therapy during the procedure. In the univariate analysis, patients with early or late transient IEs presented more often with a relative delay of venous filling during the BOT of $\geq 2 \mathrm{sec}-$ onds $(P=.043)$. This association persisted in the group of patients with late IEs $(P=.072)$

\section{Symptoms of Mass Effect}

Pain partially or completely improved in 42/43 (98\%) patients. The cranial nerve palsy partially or completely improved in $27 / 32$ (84\%) patients. Among these patients, 4/32 (12\%) presented with an initial improvement of the cranial nerve palsy, followed by an unexplained late recurrence with a time lapse ranging from 4 months to 4 years, whereas the volume of the aneurysmal sac was retracted or stable on MR imaging. The occurrence or worsening of cranial nerve palsy was observed in $2 / 56(3.5 \%)$ patients in the early follow-up after carotid occlusion. For both of these patients, it was transient in the days following the treatment and then partially or completely improved. Two other patients who did not present with any improvement of the palsy developed a corneal ulceration in the months following the carotid occlusion, related to a sensitive deficiency in the V1 trigeminal branch area already present before the procedure.

\section{Aneurysm Follow-Up}

An aneurysmal retraction was observed on MR imaging in 39/43 (91\%) patients (Fig 1D). The 4 patients without significant retraction presented with an aneurysm still circulating in a retrograde manner on the angiographic control, supplied in 3 patients by the ophthalmic artery. The absence of significant retraction was associated with a more frequent para-/supraclinoid topography of the aneurysm (3/12 [25\%] versus $1 / 31$ [4\%], $P=0.059)$. One patient of $43(2 \%)$ presented with growth of a contralateral carotid aneurysm 2 years after the carotid occlusion, requiring selective embolization with no clinical consequences.

\section{DISCUSSION}

Our study showed that in a retrospective series of 56 consecutive patients, PAO by using coils for large/giant or fusiform aneurysms of the carotid siphon presents a morbidity risk of $5 \%(2.5 \%$ in patients with infraclinoid aneurysm), a zero mortality risk, an aneurysmal retraction rate of $91 \%$, an improvement in pain of $98 \%$, and an improvement rate for cranial nerve palsy of $72 \%$ for a median follow-up of $>3$ years. Factors favoring a postprocedural ischemic complication are the para-/supraclinoid topography of the aneurysm and the presence of at least 1 acquired cardiovascular risk factor. On the contrary, the presence of a mural thrombus is associated with a decreasing ischemic risk. This is the largest published series of carotid occlusion by using coils for this type of indication, to our knowledge.

Our study shows a zero mortality rate and morbidity exclusively due to cerebral infarction immediately after the procedure without hemorrhage, supporting the results of previous series of PAO. ${ }^{3,9,11}$

The ischemic events after treatment in our series were most often due to embolism, because a majority (78\%) occurred in patients with a very good circle of Willis. The greater proportion of IEs in the group of patients presenting with a para-/ supraclinoid aneurysm could be explained by residual intracarotid flow phenomena coming from the ophthalmic artery or from external carotid emboli. Unlike in the study of Clarençon et $\mathrm{al}^{9}{ }^{9}$ the presence of a mural thrombus was associated with a decreasing ischemic risk. A hypothesis could be that the flow is already slowed down in thrombosed aneurysms and the occlusion is, therefore, more rapidly effective with a lower risk of embolus. However, no protective effect of the antiplatelet therapy during the procedure was demonstrated in our study.

The IEs occurring in the hemodynamic context and/or in pa-

AJNR Am J Neuroradiol 36:140-45 Jan 2015 www.ajnr.org 
Table 3: Comparison of main baseline characteristics in patients with and without ischemic events after carotid occlusion $(n=56)$

\begin{tabular}{lccc}
\hline \multicolumn{1}{c}{ Characteristics } & $\begin{array}{c}\text { Ischemic } \\
\text { Events }(\boldsymbol{n = 1 8 )}\end{array}$ & $\begin{array}{c}\text { No Ischemic } \\
\text { Events }(\boldsymbol{n}=\mathbf{3 8})\end{array}$ & $\boldsymbol{P}$ Value \\
\hline Age (yr) median (Q1-Q3) & $55(50-64)$ & $52(43-67)$ & .477 \\
Female (No.) (\%) & $18(100)$ & $33(86)$ & .131 \\
Vascular risk factor (No.) (\%) & $14(78)$ & $17(44)$ & .032 \\
Antiplatelet therapy (No.) (\%) & $5(28)$ & $10(36)$ & .856 \\
Giant aneurysms ( $\geq 25 \mathrm{~mm})($ No.) (\%) & $7(39)$ & $20(53)$ & .336 \\
Fusiform aneurysms (No.) (\%) & $1(6)$ & $4(10)$ & .479 \\
Infraclinoid aneurysms (No.) (\%) & $11(61)$ & $31(82)$ & .099 \\
Mural thrombus (No.) (\%) & $1(6)$ & $14(37)$ & .011 \\
Delay of phlebogram $\geq 2 \mathrm{sec}($ No.) (\%) & $4(22)$ & $3(8)$ & .140 \\
\hline
\end{tabular}

longer timeframe in which a de novo aneurysm develops after PAO, compared with the mean follow-up in our study, we cannot establish the long-term safety of PAO concerning this variable. This timeframe should probably justify life-long MR imaging surveillance for patients treated by PAO.

Whereas selective techniques with coils plus or minus non-flow-diverter stents are considered the criterion standard for the treatment of most saccular aneurysms of $<10-15 \mathrm{~mm}$, they are associated with a high rate of recanalization

tients with a longer relative delay of venous filling during BOT are most often transient and have a good prognosis. Because they can occur a long time after the PAO, they encourage preventing low flow or low blood pressure, especially during general anesthesia in patients treated despite a nonoptimal circle of Willis

The rate of IEs in our study was higher than the rate reported in most of the studies of PAO in the literature, which varies from $4.5 \%$ to $14 \% .^{8-11}$ The systematic inclusion of consecutive patients obtained from a prospective registry and the consideration of even early and late transient cerebral or retinal IEs in our study may explain a part of this difference. Besides, we cannot exclude the effect of the embolization technique performed exclusively by using coils in our series and exclusively or mostly by using detachable balloons in other series. We could hypothesize that the slower occlusion of the carotid artery (in approximately 10 minutes) obtained by using coils compared with the immediate occlusion obtained with a balloon could promote the migration of emboli. A possible improvement of PAO by using coils would be to perform a transitory occlusion with a balloon of the cervical carotid artery during the coiling. Even if most of the IEs had a good prognosis at the last follow-up-especially for the infraclinoid aneurysmstheir relatively high rate of occurrence requires restricting the indications for PAO to patients with debilitating pain, cranial nerve palsy, and/or intradural aneurysms. ${ }^{10}$

PAO in our study had an excellent efficacy for pain, a partial efficacy for cranial nerve palsy, and an excellent rate of aneurysm occlusion. These results are comparable with those in other series of PAO by using predominantly detachable balloons. ${ }^{3,9,11}$ We report 4 cases of late recurrence of oculomotor disorders despite an initial regression and a significant retraction of the aneurysm on MR imaging. This phenomenon has already been described for carotid siphon aneurysms treated by a reconstructive method by using coils. ${ }^{18}$ It has never been reported, to our knowledge, after $\mathrm{PAO}$ and remains unexplained.

We also report a case of a growing contralateral carotid aneurysm 2 years after PAO, which required coil embolization. A recent meta-analysis showed that the development of a new aneurysm or the growth of a pre-existing aneurysm after carotid occlusion has a $4.3 \%$ frequency and occurs within an average of 9.1 years after the occlusion. ${ }^{19}$ This phenomenon would be promoted by the high compensatory flow of the substitute pathways of the occluded carotid artery. This risk is low but higher than the risk reported for reconstructive treatments. ${ }^{20}$ Given the expected in larger aneurysms. ${ }^{4,5,11}$ Endovascular PAO with trapping has long been considered the first-line treatment of large aneurysms. New reconstructive stent-assisted treatments with flow diverters are increasingly being developed because they have been shown to be effective in several cases series. A recent prospective noncomparative study including 107 patients treated with Pipeline flow diverters (Covidien) for large or giant aneurysms of the carotid siphon showed a complete aneurysmal occlusion rate of $73.6 \%$ at 180 days and a rate of major ipsilateral stroke or neurologic death at 180 days of $5.6 \%$. The safety after 180 days and changes in the symptoms of mass effect were not available. ${ }^{14}$ One other noncomparative study, including 27 patients, reported excellent flow-diverter stenting of aneurysms with symptoms of mass effect, with a $100 \%$ rate of improvement of the symptoms of mass effect and of aneurysm retraction. ${ }^{12}$ Because they preserve the parent vessel, flow-diverter stents might prevent the occurrence of an early or late hemodynamic ischemic event and de novo aneurysms. Nevertheless, the superiority of this technique has not yet been proved by comparative studies with PAO and is still debated. ${ }^{21}$ Furthermore, recent meta-analyses concerning flow-diverter stents show that the mortality rate is close to $4 \%$, regardless of the type of aneurysm. ${ }^{22,23}$ It was $4 \%$ in the study of Briganti et $\mathrm{al}^{23}$ for 76 patients treated for intracavernous aneurysms. The reported fatal complications with stent-assisted techniques are due to major hemorrhagic or ischemic complications, which are completely absent from carotid occlusion series.

Almost one-fifth patients in our study were lost to follow-up. They were almost exclusively patients living abroad for whom the follow-up could not be performed in our hospital or in a reference center in their country and whose phone numbers were unavailable or incorrect. It is very unlikely that taking these into account would have modified the morbidity and mortality rates because serious complications were observed only during the early followup, which could be analyzed for each patient. Furthermore, baseline characteristics were similar in patients with and without a late follow-up. Given the exploratory nature of this study, blind analysis by an independent review board has not been performed, introducing potential interpretation biases.

\section{CONCLUSIONS}

In this study, PAO to treat large, giant, or fusiform aneurysms of the carotid siphon is safe and effective, especially for giant infraclinoid aneurysms. Because of the ischemic event risk, this treatment should be limited to symptomatic patients or aneurysms 
with a high risk of severe bleeding. According to recent metaanalyses, our results suggest that the validation of stent-assisted reconstructive treatments for these aneurysms requires controlled studies with PAO.

\section{ACKNOWLEDGMENTS}

The authors would like to thank the Head and Neck Department at the Lariboisière Hospital for their direct or indirect intervention in the management of our study patients.

\section{REFERENCES}

1. Goldenberg-Cohen N, Curry C, Miller NR, et al. Long term visual and neurological prognosis in patients with treated and untreated cavernous sinus aneurysms. J Neurol Neurosurg Psychiatry 2004;75: 863-67

2. Barrow DL, Alleyne C. Natural history of giant intracranial aneurysms and indications for intervention. Clin Neurosurg 1995;42: $214-44$

3. Zhang Z, Lv X, Wu Z, et al. Clinical and angiographic outcome of endovascular and conservative treatment for giant cavernous carotid artery aneurysms. Interv Neuroradiol 2014;20:29-36

4. Gao X, Liang G, Li Z, et al. A single-centre experience and follow-up of patients with endovascular coiling of large and giant intracranial aneurysms with parent artery preservation. J Clin Neurosci 2012; 19:364-69

5. van Rooij WJ, Sluzewski M. Unruptured large and giant carotid artery aneurysms presenting with cranial nerve palsy: comparison of clinical recovery after selective aneurysm coiling and therapeutic carotid artery occlusion. AJNR Am J Neuroradiol 2008;29:997-1002

6. Chalouhi N, Tjoumakaris S, Gonzalez LF, et al. Coiling of large and giant aneurysms: complications and long-term results of 334 cases. AJNR Am J Neuroradiol 2014;35:546-52

7. Larson JJ, Tew JM Jr, Tomsick TA, et al. Treatment of aneurysms of the internal carotid artery by intravascular balloon occlusion: longterm follow-up of 58 patients. Neurosurgery 1995;36:26-30; discussion 30

8. van Rooij WJ. Endovascular treatment of cavernous sinus aneurysms. AJNR Am J Neuroradiol 2012;33:323-26

9. Clarençon F, Bonneville F, Boch AL, et al. Parent artery occlusion is not obsolete in giant aneurysms of the ICA: experience with verylong-term follow-up. Neuroradiology 2011;53:973-82

10. Stiebel-Kalish H, Kalish Y, Bar-On RH, et al. Presentation, natural history, and management of carotid cavernous aneurysms. Neurosurgery 2005;57:850-57; discussion 850-57
11. van der Schaaf IC, Brilstra EH, Buskens E, et al. Endovascular treatment of aneurysms in the cavernous sinus: a systematic review on balloon occlusion of the parent vessel and embolization with coils. Stroke 2002;33:313-18

12. Szikora I, Marosfoi M, Salomvary B, et al. Resolution of mass effect and compression symptoms following endoluminal flow diversion for the treatment of intracranial aneurysms. AJNR Am J Neuroradiol 2013;34:935-39

13. Chalouhi N, Tjoumakaris S, Starke RM, et al. Comparison of flow diversion and coiling in large unruptured intracranial saccular aneurysms. Stroke 2013;44:2150-54

14. Becske T, Kallmes DF, Saatci I, et al. Pipeline for uncoilable or failed aneurysms: results from a multicenter clinical trial. Radiology 2013;267:858-68

15. Abud DG, Spelle L, Piotin M, et al. Venous phase timing during balloon test occlusion as a criterion for permanent internal carotid artery sacrifice. AJNR Am J Neuroradiol 2005;26:2602-09

16. Struffert T, Deuerling-Zheng Y, Engelhorn T, et al. Monitoring of balloon test occlusion of the internal carotid artery by parametric color coding and perfusion imaging within the angio suite: first results. Clin Neuroradiol 2013;23:285-92

17. Gevers S, Heijtel D, Ferns SP, et al. Cerebral perfusion long term after therapeutic occlusion of the internal carotid artery in patients who tolerated angiographic balloon test occlusion. AJNR Am JNeuroradiol 2012;33:329-35

18. Dagain A, Nataf F, Page P, et al. Endovascular coil transfixing a cranial nerve five years after embolisation. Acta Neurochir (Wien) 2008;150:705-07; discussion 707

19. Arambepola PK, McEvoy SD, Bulsara KR. De novo aneurysm formation after carotid artery occlusion for cerebral aneurysms. Skull Base 2010;20:405-08

20. Ferns SP, Sprengers ME, van Rooij WJ, et al. De novo aneurysm formation and growth of untreated aneurysms: a 5-year MRA follow-up in a large cohort of patients with coiled aneurysms and review of the literature. Stroke 2011;42:313-18

21. van Rooij WJ, Sluzewski M. Unruptured carotid artery aneurysms presenting with symptoms of mass effect: outcome after selective coiling, parent vessel occlusion, and flow diversion. AJNR Am J Neuroradiol 2013;34:940-41

22. Brinjikji W, Murad MH, Lanzino G, et al. Endovascular treatment of intracranial aneurysms with flow diverters: a meta-analysis. Stroke 2013;44:442-47

23. Briganti F, Napoli M, Tortora F, et al. Italian multicenter experience with flow-diverter devices for intracranial unruptured aneurysm treatment with periprocedural complications: a retrospective data analysis. Neuroradiology 2012;54:1145-52 